\title{
Correlation between Gamma-ray Sky and Catalogs of Galaxies and Galaxy Clusters
}

\section{Marco Regis*}

Dipartimento di Fisica, Università di Torino, and Istituto Nazionale di Fisica Nucleare, Sezione di Torino, via P. Giuria 1, I-10125 Torino, Italy

E-mail: regisato.infn.it

We discuss here methods and results concerning the angular cross-correlation between gammaray sky-maps and catalogs of galaxies and galaxy clusters. The main goal of the study is to extract information on the clustering, redshift distribution and type of the unresolved non-thermal gamma-ray sources. We will show how this technique can be used to search for "new" populations of sources, including galaxy clusters and the one possibly provided by annihilation or decay of particle dark matter.

XII Multifrequency Behaviour of High Energy Cosmic Sources Workshop

12-17 June

Palermo, Italy

${ }^{*}$ Speaker. 
Let us consider two populations ( $a$ and $b$ ) of astrophysical sources. The probability of finding a pair with an object from population $a$ in a volume $d V_{1}$ of the Universe and an object from population $b$ in $d V_{2}$ is given by [1]: $d P=\rho_{a} d V_{1} \rho_{b} d V_{2}\left(1+\xi_{a b}\left(\vec{r}_{12}\right)\right)$, where $\xi_{a b}$ gives the excess probability as a function of $\vec{r}_{12}$ (i.e., $\xi_{a b}=0$ for Gaussian random fields). On the other hand, we can also write the probability as $d P=\left\langle\rho_{a}\left(\vec{x}_{1}\right) d V_{1} \rho_{b}\left(\vec{x}_{1}+\vec{r}_{12}\right) d V_{2}\right\rangle$, where $\langle.$.$\rangle denotes ensemble average.$ With a few simple steps (and considering $\delta(\vec{x})=\rho(\vec{x}) /\langle\rho\rangle-1$ ), we arrive to the definition of the two-point correlation function:

$$
\xi_{a b}(r)=\left\langle\delta_{a}\left(x_{1}\right), \delta_{b}\left(x_{1}+r\right)\right\rangle_{V}
$$

where we assumed our Universe to satisfy statistical homogeneity (and thus $\xi$ depends only on the modulus of $\vec{r}$ ) and ergodicity (to perform a volume average instead of an ensemble average).

In the works discussed here, we measure the angular cross-correlation between different sky maps and therefore $a$ and $b$ are points in two different maps at different wavelengths. The twopoint cross-correlation function projected on the sky is named $C C F^{(a b)}(\theta)$. It is sometime more convenient to work in the harmonic space (see, e.g., [2]), deriving the cross-correlation angular power spectrum (CAPS) $C_{\ell}^{a b}$. The latter is relate to the $C C F$ via a Legendre transformation:

$$
C C F^{(a b)}(\theta)=\sum_{\ell} \frac{2 \ell+1}{4 \pi} C_{\ell}^{a b} P_{\ell}[\cos (\theta)] .
$$

The general idea is to use maps of known objects (galaxies, galaxy clusters, matter distribution) to isolate contributions from certain populations (in particular, galaxy clusters and particle dark matter) in the unresolved background of maps at different wavelengths.

In Ref. [3], the detection of a cross-correlation signal between FermiLarge Area Telescope diffuse $\gamma$-ray maps and catalogs of clusters was reported. The analysis involved three different catalogs: WHL12, redMaPPer and PlanckSZ. They all show a positive correlation with different amplitudes, see Fig. 1. The signal detection is confirmed by the results of a stacking analysis. The cross-correlation signal extends to rather large angular scales, around 1 degree, that correspond, at the typical redshift of the clusters in these catalogs, to a few to tens of $\mathrm{Mpc}$, i.e. the typical scale-length of the large scale structures in the Universe. Most likely this signal is contributed by the cumulative emission from AGNs associated to the filamentary structures that converge toward the high peaks of the matter density field in which galaxy clusters reside. In addition, our analysis reveals the presence of a second component, more compact in size and compatible with a point-like emission from within individual clusters. At present, we cannot distinguish between the two most likely interpretations for such a signal, i.e. whether it is produced by AGNs inside clusters or if it is a diffuse $\gamma$-ray emission from the intra-cluster medium. This latter, intriguing, hypothesis might be tested by applying this technique to a low redshift large mass cluster sample.

One of the most relevant applications of the cross-correlation analysis is related to the search for particle dark matter (DM). Indeed, even if DM halos are too faint to be individually detected in $\gamma$-rays, they form the most numerous population in the Universe. Therefore, the DM "cumulative" signal and its spatial coherence might be observable. The cross-correlation of $\gamma$-rays with gravitational tracers has been shown to greatly enhance the possibility to infer informations about extragalactic DM with respect to $\gamma$-rays alone (see, e.g., [2]). One of the most important aspects 

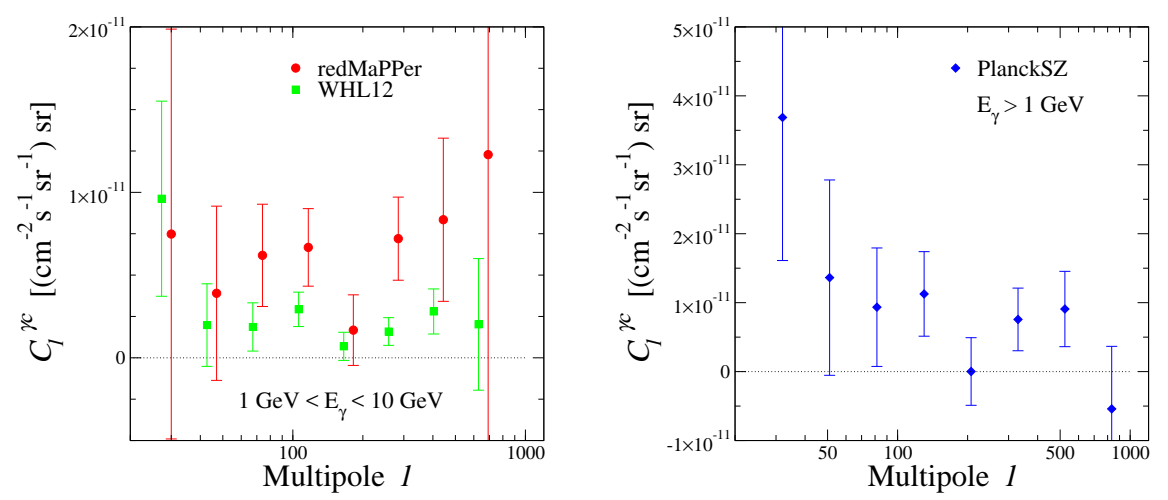

Figure 1: Left panel: Observed CAPS (PSF deconvolved) between the Fermi-LAT $\gamma$-ray map and the redMaPPer (red) and WHL12 (green) catalogs of clusters at $1 \mathrm{GeV}<E_{\gamma}<10 \mathrm{GeV}$. Right panel: Observed CAPS between the Fermi-LAT $\gamma$-ray map at $E_{\gamma}>1 \mathrm{GeV}$ and the PlanckSZ catalog. Figures taken from [3].

of this technique is the possibility of performing a tomographic approach (not possible with $\gamma$-ray signals alone), using the redshift information associated to the gravitational tracers. The fact that the redshift evolution of the WIMP source is predicted to be very different from other astrophysical non-thermal emitters can be used to disentangle a DM signal in these channels.

In Refs. [4, 5], we compare the measured angular cross-correlation between the Fermi-LAT $\gamma$-ray sky and catalogs of extra-galactic objects with the expected signal induced by weakly interacting massive particle (WIMP) DM. We include also a detailed description of the contribution of astrophysical $\gamma$-ray emitters such as blazars, misaligned AGN and star forming galaxies, and perform a global fit to the measured cross-correlation. Five catalogs are considered: SDSS-DR6 quasars, 2MASS galaxies, NVSS radio galaxies, SDSS-DR8 Luminous Red Galaxies and SDSSDR8 main galaxy sample. To model the cross-correlation signal we use the halo occupation distribution formalism to estimate the number of galaxies of a given catalog in DM halos and their spatial correlation properties. The use of different catalogs probing objects at different redshifts reduces significantly, though not completely, the degeneracy among the different $\gamma$-ray components. The presence of a significant WIMP DM signal is allowed by the data but not significantly preferred by the fit, due to a degeneracy with the misaligned AGN component. Assuming modest substructure boost, the analysis excludes thermal annihilation cross sections at 95\% level for WIMP masses up to few tens of $\mathrm{GeV}$. Constraining the low-redshift properties of astrophysical populations with future data will further improve the sensitivity to DM.

Summarizing, we argued that angular cross-correlations of Fermi-LAT maps with catalogs of galaxies and galaxy clusters are a powerful way to detect faint $\gamma$-ray populations, such as the intra-cluster medium and particle DM.

M.R. acknowledges support by the Excellent Young PI Grant: The Particle Dark-matter Quest in the Extragalactic Sky funded by the University of Torino and Compagnia di San Paolo, and by the research grant TAsP (Theoretical Astroparticle Physics) funded by the Istituto Nazionale di Fisica Nucleare (INFN). 

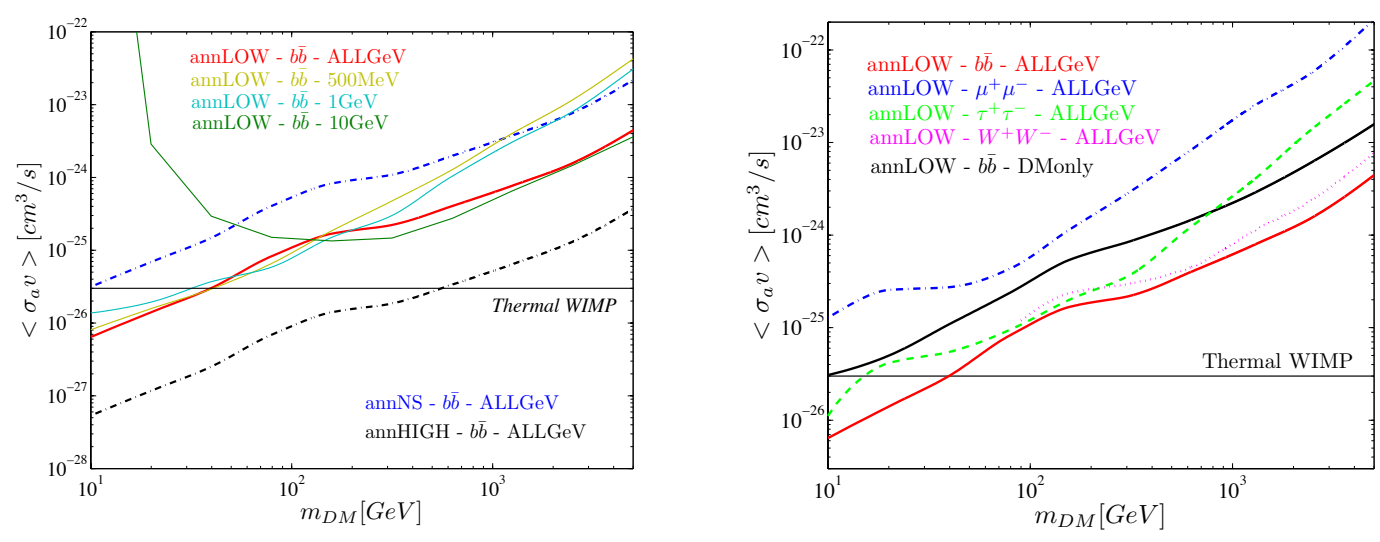

Figure 2: $95 \%$ upper bounds on the DM annihilation rate $\sigma_{a} v$ as a function of the DM mass. Figure taken from [5], to which we refer the reader for details.

\section{DISCUSSION}

CARLOTTA PITTORI: Why do you select only Femi-LAT data above $500 \mathrm{MeV}$ (with Pass8)?

MARCO REGIS: This is because the Fermi-LAT PSF below this energy is quite large, and once you have masked all the known sources, the fraction of the sky available to perform the crosscorrelation analysis becomes too small.

\section{References}

[1] Peacock, J. A., Cosmological Physics, Cambridge University Press, 1999, 704.

[2] N. Fornengo and M. Regis, Front. Physics 2 (2014) 6 doi:10.3389/fphy.2014.00006 [arXiv:1312.4835 [astro-ph.CO]].

[3] E. Branchini, S. Camera, A. Cuoco, N. Fornengo, M. Regis, M. Viel and J. Q. Xia, Astrophys. J. Suppl. 228 (2017) no.1, 8 doi:10.3847/1538-4365/228/1/8 [arXiv:1612.05788 [astro-ph.CO]].

[4] M. Regis, J. Q. Xia, A. Cuoco, E. Branchini, N. Fornengo and M. Viel, Phys. Rev. Lett. 114 (2015) no.24, 241301 doi:10.1103/PhysRevLett.114.241301 [arXiv:1503.05922 [astro-ph.CO]].

[5] A. Cuoco, J. Q. Xia, M. Regis, E. Branchini, N. Fornengo and M. Viel, Astrophys. J. Suppl. 221 (2015) no.2, 29 doi:10.1088/0067-0049/221/2/29 [arXiv:1506.01030 [astro-ph.HE]]. 